\title{
LOCAL COMPLEX ANALYTIC CURVES IN AN ANALYTIC VARIETY
}

\author{
BERNARD SHIFFMAN ${ }^{1}$
}

1. Introduction. In a recent paper [3], H. Whitney posed the following problems:

Let $X$ be a complex analytic variety, and let $y$ be a nonisolated point of $X$.

(1) Can we choose a neighborhood $N$ of $y$ such that for each point $x$ of $N$ there exists a connected one-dimensional complex analytic subvariety $C(x)$ of $N$ that contains $x$ and $y$ and is regular at each of its points except possibly $y[3$, p. 214]?

(2) Furthermore, can we choose the one-dimensional subvarieties $C(x)$ as above so that any two distinct members of the family $\{C(x)\}$ intersect only at $y[3$, p. 231]?

In this paper, we give a short elementary solution to problem (1), using some methods of T. Bloom [1]. The solution to problem (2) with $\operatorname{dim} X=2$ is a special case of a result of Bloom [1]. We do not know the answer to question (2) with $\operatorname{dim} X>2$.

We use the following terminology:

An analytic set in a (reduced) complex analytic space $X$ is a closed complex analytic subvariety of $X$. An analytic set is said to be regular if it contains no singular points (i.e., if it is a complex manifold). An analytic curve in $X$ is a pure 1-dimensional analytic set in $X$; an analytic hypersurface is an analytic set of constant codimension 1 . Other standard terminology used in this paper can be found in [2].

TheOREM 1. Let $X$ be a complex analytic space, $Y$ an analytic set in $X$, and $y$ a point of $Y$. Then there exists a neighborhood $N$ of $y$ in $X$ such that for all $x \in N-Y$, we can find a (globally) irreducible analytic curve $C(x)$ in $N$ such that

(i) $x \in C(x)$,

(ii) $C(x) \cap Y=\{y\}$,

(iii) $C(x)-\{y\}$ is regular.

The affirmative answer to question (1) follows from Theorem 1 with $Y=\{y\}$. Note that it does not follow that $C(x)$ is locally irreducible at $y$, and it remains an open question whether we can extend Theorem 1 by adding the condition that $C(x)$ be irreducible at $y$.

Received by the editors May 19, 1969.

1 This research was carried out in 1967, while the author held a National Science Foundation Graduate Fellowship at the University of California at Berkeley. 
We shall show that Theorem 1 is a consequence of the following result.

Theorem 2. Let $U$ be an open set in $C^{n+1}(n \geqq 1)$, and let $A$ be an analytic hypersurface in $U$ such that $0 \in A$. Then there exists a neighborhood $U^{\prime} \subset U$ of 0 such that for all $p \in U^{\prime}-A$, we can find a connected regular analytic curve $C(p)$ in $U^{\prime}$ with $p \in C(p)$ and $C(p) \cap A=\{0\}$.

2. Proof that Theorem 2 implies Theorem 1 . We use induction on $\operatorname{dim}_{y} X$. If $\operatorname{dim}_{y} X$ is 0 or 1 , the theorem is trivial. So suppose Theorem 1 has been proven for dimensions up to and including $n$, and let $\operatorname{dim}_{y} X=n+1$. We assume without loss of generality that $\operatorname{dim}_{y} Y \leqq n$, since otherwise we can ignore those irreducible $(n+1)$-dimensional components of $X$ which are contained in $Y$. By restricting our consideration to a sufficiently small neighborhood of $y$ (which we also call $X$ ), we can assume that $X$ has the following "local parametrization": $X=X_{1} \cup Z$, where $Z$ is an analytic variety of dimension at most $n$ containing $y$, and $X_{1}$ is an analytic variety of pure dimension $n+1$ containing $y$; we furthermore have a neighborhood $U$ of 0 in $C^{n+1}$, an analytic hypersurface $A$ in $U$, and a proper holomorphic map $\pi: X_{1} \rightarrow U$ such that $\pi^{-1}(0)=\{y\}, \pi\left(X_{1} \cap Z\right) \subset A$, and $\pi$ is a local biholomorphism outside of $\pi^{-1}(A)$.

Note that $0=\pi(y) \in A$ since $y \in X_{1} \cap Z$. Let

$$
T=\pi^{-1}(A) \subset X_{1}, \quad X_{2}=T \cup Z .
$$

Therefore $X_{1} \cup X_{2}=X, X_{1} \cap X_{2}=T$, and $\operatorname{dim} X_{2}=n$. Choose a neighborhood $U^{\prime} \subset U$ of 0 as in the statement of Theorem 2, and let

$$
N_{1}=\pi^{-1}\left(U^{\prime}\right) \subset X_{1} \text {. }
$$

Choose an open $W$ in $X$ such that $N_{1}=X_{1} \cap W$. By the induction hypothesis, we can choose a neighborhood $N_{2} \subset X_{2} \cap W$ of $y$ that satisfies the conditions of Theorem 1 applied to $X_{2} \cap W$. Let

$$
N=\left(N_{1}-T\right) \cup N_{2}=W-\left(X_{2}-N_{2}\right),
$$

which is open in $X$ and contains $y$.

In order to show that $N$ satisfies the conditions of Theorem 1, it suffices to consider $x \in N_{1}-T \subset N_{1}-Y$. Then $\pi(x) \in U^{\prime}-A$. Let $L$ be a connected regular analytic curve in $U^{\prime}$ with $\pi(x) \in L$ and $L \cap A=\{0\}$, and let $C^{\prime}=\pi^{-1}(L) \subset N_{1}$. Since $C^{\prime} \cap T=\pi^{-1}(0)=\{y\}$, it follows that $C^{\prime}$ is an analytic curve in $N, C^{\prime}-\{y\}$ is regular, and $C^{\prime} \cap Y=\{y\}$. Let $C(x)$ be the irreducible component of $C^{\prime}$ that contains $x$. Since $L$ is irreducible, $\pi(C(x))=L$ and therefore $y \in C(x)$. Thus $C(x)$ is our desired analytic curve. 
3. Proof of Theorem 2. We adopt the following convention throughout this section: If $\xi$ is either a point in $\boldsymbol{C}^{n+1}$ or a function with values in $\boldsymbol{C}^{n+1}$, we write $\xi=\left(\xi^{0}, \xi^{1}, \cdots, \xi^{n}\right)$.

Let $A$ and $U$ be given as in the statement of Theorem 2. Shrink $U$ if necessary, so that we can choose a holomorphic function $f$ on $U$ such that

$$
A=\operatorname{loc}(f)=\{z \in U: f(z)=0\} .
$$

Make a linear change of coordinates (if necessary) so that 0 is an isolated point of $\operatorname{loc}\left(f, z^{1}, \cdots, z^{n}\right)$, where $z^{0}, z^{1}, \cdots, z^{n}$ are the coordinates in $U \subset C^{n+1}$. Let

$$
\pi=\left(f, z^{1}, \cdots, z^{n}\right): U \rightarrow C^{n+1} .
$$

Choose a neighborhood $V \subset C^{n+1}$ of 0 and shrink $U$ again so that $\pi(U) \subset V, \pi: U \rightarrow V$ is a proper map, and $\pi^{-1}(0)=\{0\}$ (see $[2$, p. $161])$. Assume that $\partial f / \partial z^{0}$ vanishes at 0 , since otherwise the conclusion of Theorem 2 would be obvious. Then $\operatorname{loc}\left(\partial f / \partial z^{0}\right)$ is an analytic hypersurface in $U$ containing 0 . Let $B=\pi\left(\operatorname{loc}\left(\partial f / \partial z^{0}\right)\right)$, an analytic hypersurface in $V$. Choose a connected neighborhood $V^{\prime} \subset V$ of 0 and a holomorphic function $h$ on $V^{\prime}$ such that $B \cap V^{\prime}=\operatorname{loc}(h)$. Choose open balls $\Delta$ and $\Delta^{\prime}$ about 0 such that $\Delta^{\prime} \subset \subset \Delta \subset \subset V^{\prime}$. Let $U^{\prime}=\pi^{-1}\left(\Delta^{\prime}\right)$, and note that

$$
A \cap U^{\prime}=\pi^{-1}\left\{w \in \Delta^{\prime}: w^{0}=0\right\} .
$$

Let $p$ be an arbitrary point in $U^{\prime}-A$. Let $a=\pi(p) \in \Delta^{\prime}$ (note that $a^{0} \neq 0$ ), and let $L_{a} \subset C^{n+1}$ be the complex line containing 0 and $a$. (The family of analytic curves $\left\{\pi^{-1}\left(L_{a} \cap \Delta^{\prime}\right)\right\}$ is a special case of a construction of Bloom [1].) In order to modify $L_{a}$ so that we obtain (via $\pi^{-1}$ ) a regular analytic curve in $U^{\prime}$ that satisfies the conditions of the theorem, we need the following definition and lemma (which is proved at the end of this section).

Definition. If $f$ is a holomorphic function defined in a neighborhood of a point $x \in C^{m}$, we let $\nu(f ; x)$ denote the order of $f$ at $x(\nu(f ; x)$ $=0$ if $f(x) \neq 0 ; \nu(f ; x)=+\infty$ if $f \equiv 0)$. Let $h \neq 0$ be a holomorphic function on a connected open set $V \subset C^{n+1}$. Let $K$ be a closed subset of $C$ without isolated points, and let $g: K \rightarrow V$ be holomorphic (i.e., $g$ can be extended holomorphically to a neighborhood of $K$ ). We say that $g$ is h-transverse if

$$
\nu(h \circ g ; t)=\nu(h ; g(t)) \quad \text { for all } t \in K .
$$

(The condition that $g$ be $h$-transverse means geometrically that for each point $t_{0} \in \operatorname{loc}(h \circ g)$, the image under $g_{*}$ of the tangent space of 
$C$ at $t_{0}$ is not contained in the tangent cone [3, pp. 211, 219-223] of loc $(h)$ at $g\left(t_{0}\right)$.)

LEMma. Let $h \not \equiv 0$ be a holomorphic function on a connected open set $V \subset C^{n+1}$, and let $K$ be a connected compact subset of $C$. Let $c_{1}$ and $c_{2}$ be distinct points of $K$, and let $w_{1}, w_{2} \in V$. Consider the metric space $\mathcal{F}$ (with the sup-norm metric) of all holomorphic maps $g=\left(g^{0}, \cdots, g^{n}\right): K \rightarrow V$ such that $g^{0}(t)=t$ and $g\left(c_{j}\right)=w_{j}$ for $j=1,2$. Suppose that $\mathcal{F}$ is not empty. Then the set of h-transverse maps in $\mathcal{F}$ is dense in $\mathcal{F}$.

Let $\psi: C \rightarrow C^{n+1}$ be the linear map given by $\psi\left(a^{0}\right)=a$ (and thus Image $\left.(\psi)=L_{a}\right)$. Let $K=\psi^{-1}(\bar{\Delta})$, a closed disk about 0; let

$$
J=\left\{r a^{0}: 0 \leqq r \leqq 1\right\} \subset K .
$$

By applying the above lemma (with $c_{1}=0, c_{2}=a^{0}, w_{1}=0, w_{2}=a$ ), we can choose an $h$-transverse holomorphic map $g: K \rightarrow V^{\prime}($ near $\psi \mid K)$ such that

(1) $g^{0}(t)=t$,

(2) $g(0)=0, g\left(a^{0}\right)=a$,

(3) $g(J) \subset \Delta^{\prime}$,

(4) $g(\partial K) \subset V^{\prime}-\bar{\Delta}^{\prime}$.

Let $t_{1}, \cdots, t_{m} \in K$ be the distinct zeros of $h \circ g$, and let $x_{j}=g\left(t_{j}\right) \in V^{\prime}$, for $1 \leqq j \leqq m$. Let $\gamma(t)$ be a polynomial which vanishes to first order at $t_{1}, \cdots, t_{m}$, and $a^{0}$. (Note that $h \circ g(0)=0$, so one of the $t_{j}$ must be 0 .) For $\lambda=\left(0, \lambda^{1}, \cdots, \lambda^{n}\right) \in C^{n+1}$, define

$$
g_{\lambda}(t)=g(t)+\gamma(t) \lambda .
$$

For $\lambda$ sufficiently small,

$$
\nu\left(h \circ g_{\lambda} ; t_{j}\right) \geqq \nu\left(h ; x_{j}\right)=\nu\left(h \circ g ; t_{j}\right),
$$

and therefore $\nu\left(h \circ g_{\lambda} ; t_{j}\right)=\nu\left(h \circ g ; t_{j}\right)$ for $1 \leqq j \leqq m$, and $t_{1}, \cdots, t_{m}$ are the only zeros of $g_{\lambda}$ in $K$. Thus, for such $\lambda$,

$$
g_{\lambda}(K) \cap B=\left\{x_{1}, \cdots, x_{m}\right\} .
$$

Let $C_{\lambda}=\pi^{-1}\left(g_{\lambda}(K) \cap \Delta^{\prime}\right)$. For $\lambda$ small, $C_{\lambda}$ is an analytic curve in $U^{\prime}$ that is regular outside of the finite set $S=\pi^{-1}\left\{x_{1}, \cdots, x_{m}\right\}$, since $\pi$ has rank $n+1$ wherever $\partial f / \partial z^{0} \neq 0$. Consider an arbitrary point $q \in S \cap U^{\prime}$. Let

$$
\left.\frac{\partial f}{\partial z^{k}}\right|_{q}=\beta_{k},\left.\quad \frac{\partial g_{\lambda}^{k}}{\partial t}\right|_{\tau(q)}=\alpha_{\lambda}^{k}, \quad \text { for } 1 \leqq k \leqq n .
$$

Since 


$$
C_{\lambda}=\operatorname{loc}\left(z^{1}-g_{\lambda}^{1} \circ f, \cdots, z^{n}-g_{\lambda}^{n} \circ f\right) \cap U^{\prime},
$$

it follows that $C_{\lambda}$ is regular at $q$ provided that the determinant

$$
d=\operatorname{det}\left(\delta_{j}^{k}-\beta_{j} \alpha_{\lambda}^{k}\right) \quad(1 \leqq j, k \leqq n)
$$

does not vanish (where $\delta_{j}^{k}=1$ if $k=j, \delta_{j}^{k}=0$ if $k \neq j$ ). A simple calculation (for example, consider the characteristic polynomial of the matrix $\left.\left(\beta_{j} \alpha_{\lambda}^{k}\right)\right)$ shows that

$$
d=1-\sum_{1}^{n} \beta_{k} \alpha_{\lambda}^{k}
$$

Since $S$ is finite, we conclude that we can choose an arbitrarily small $\lambda=\left(0, \lambda^{1}, \cdots, \lambda^{n}\right)$ such that $C_{\lambda}$ is regular. (One can also arrive at this conclusion, without calculating determinants, by instead proving a general fact about holomorphic maps from $U \subset C^{n+1}$ into $C^{n+1}$ that have rank $n$ at a given point $q \in U$.) Choose a small $\lambda$ such that $g_{\lambda}$ satisfies conditions (1) through (4) above and $C_{\lambda}$ is a regular analytic curve. Let $C(p)$ be the connected component of $C_{\lambda}$ that contains $p$. Therefore, the analytic curve $\pi(C(p))$ equals the connected component of $g_{\lambda}(K) \cap \Delta^{\prime}$ that contains $a$. Condition (3) above then implies that $0 \in \pi(C(p))$, and therefore $0 \in C(p)$. Thus $C(p)$ is our desired analytic curve.

To complete this discussion, we now prove the lemma: Let $\mathcal{F}, h$, etc., be given as in the statement of the lemma. For $f \in \mathcal{F}$, define

$$
\begin{aligned}
I(f ; t) & =\nu(h \circ f ; t)-\nu(h ; f(t)) \geqq 0, \\
I(f) & =\sum I(f ; t) \quad(t \in K) .
\end{aligned}
$$

(The above sum is finite if $h \circ f \not \equiv 0 ; I(f)=+\infty$ if $h \circ f \equiv 0$.) Let $\mathcal{F}_{0}$ be an arbitrary nonempty open subset of $\mathcal{F}$. Choose a function $g \in \mathcal{F}_{0}$ such that

$$
I(g)=\min \left\{I(f): f \in \Im_{0}\right\} .
$$

We must show that $I(g)=0$. Suppose, on the contrary, that $I(g) \geqq 1$. If $I(g)<+\infty$, let $t_{1}, \cdots, t_{m} \in K$ be the distinct zeros of $h \circ g$. Then

$$
I(g)=\sum_{1}^{m} I\left(g ; t_{j}\right) \text {. }
$$

Assume without loss of generality that $I\left(g ; t_{1}\right)>0$. Let $\gamma(t)$ be a polynomial that vanishes to first order at the points $c_{1}, c_{2}, t_{1}, \cdots, t_{m}$. For $\lambda=\left(0, \lambda^{1}, \cdots, \lambda^{n}\right) \in C^{n+1}$, define 


$$
g_{\lambda}(t)=g(t)+\gamma(t) \lambda, \quad \text { for } t \in K .
$$

Thus $g_{\lambda}\left(t_{j}\right)=g\left(t_{j}\right)$ and $g_{\lambda} \in F_{0}$ for $\lambda$ sufficiently small. By considering the Taylor expansion of $h \circ g_{\lambda}$ about $t_{1}$, we conclude that there exist arbitrarily small $\lambda=\left(0, \lambda^{1}, \cdots, \lambda^{n}\right)$ such that

$$
\nu\left(h \circ g_{\lambda} ; t_{1}\right)=\nu\left(h ; g\left(t_{1}\right)\right)<\nu\left(h \circ g ; t_{1}\right) .
$$

For such a $\lambda$ sufficiently small, we let $Z_{j} \subset K$ denote the set of zeros of $g_{\lambda}$ near $t_{j}($ for $1 \leqq j \leqq m)$, and we conclude that

$$
\sum\left(I\left(g_{\lambda} ; t\right): t \in Z_{j}\right) \leqq I\left(g ; t_{j}\right),
$$

with the strict inequality holding for $j=1$. Hence $I\left(g_{\lambda}\right)<I(g)$, contradicting the minimality of $I(g)$. Finally, if $I(g)=+\infty$, by repeating the above argument with $m=1$ and $t_{1}$ an arbitrary point of $K$, we obtain $g_{\lambda}$ with $I\left(g_{\lambda}\right)<+\infty$, also contradicting the minimality of $I(g)$.

\section{REFERENCES}

1. T. Bloom, Local fiberings of a complex analytic variety, Math. Ann. 172 (1967), 313-326. MR 36 \#4023.

2. R. Gunning and H. Rossi, Analytic functions of several complex variables, Prentice-Hall, Englewood Cliffs, N. J., 1965. MR 31 \#4927.

3. H. Whitney, Local properties of analytic varieties, Differential and Combinatorial Topology, Princeton Univ. Press, Princeton N. J., 1965, pp. 205-244. MR 32 \#5924.

Massachusetts Institute of Technology 